\title{
Interfacial interactions between nitrifying bacteria and mineral carriers in aqueous media determined by contact angle measurements and thin layer wicking
}

\author{
P. Teixeira ${ }^{a}, J$. Azeredo ${ }^{a}$, R. Oliveira ${ }^{a, *}$, E. Chibowski ${ }^{b}$ \\ a Departamento de Engenharia Biológica, Instituto de Biotecnologia e Química Fina, Universidade do Minho, Campus de Gualtar, \\ 4709 Braga codex, Portugal \\ b Department of Physical Chemistry, Faculty of Chemistry, Maria Curie-Sklodowska University, 20-031 Lublin, Poland
}

Received 20 May 1998; accepted 14 October 1998

\begin{abstract}
Contact angle measurements enable the determination of the surface free energy on flat and smooth solid surfaces. The thin layer wicking technique permits this determination for powdered materials. Both techniques were assayed on limestone and basalt which were the materials used as supports for bacterial adhesion. The resulting surface free energy components were compared. The free energy of interaction between nitrifying bacteria and support materials in aqueous medium $\left(\Delta G_{132}\right)$ was calculated and correlated with bacterial adhesion observed in a previous study. Although the values of the polar and apolar components were not exactly the same, both techniques lead to the same conclusions in terms of the ability for bacterial adhesion. (c) 1998 Elsevier Science B.V. All rights reserved.
\end{abstract}

Keywords: Contact angle; Thin layer wicking; Surface free energy

\section{Introduction}

Microbial adhesion to solid surfaces is one of the prime steps in the formation of biofilms [1]. In this process, the surface properties of both interacting bodies, such as surface charge and surface free energy play an important role. Generally, an organism tends to adsorb strongly to minimise the free energy of the system [2]. However, charge repulsion or steric exclusion may prevent a close

\footnotetext{
* Corresponding author. Fax: + 351-53-678-986; e-mail: roliveira@deb.uminho.pt.
}

approach. Hydrophilic surfaces may be stabilised by ordering the surface-associated water molecules toward the bulk phase, thus increasing their free energy. Hydrophobic surfaces should attract strongly because of the tendency to move water molecules away from the surface into the bulk. This will decrease their free energy and the level of hydrogen bonding among water molecules will increase [3]. Surface free energy considerations enable computation of the thermodynamic free energy of adhesion between two surfaces by regarding the process as a replacement of two solid-liquid interfaces by one solid-solid inter- 
face. According to this model, adhesion of bacteria will be favoured if the process itself causes a decrease of the free energy. Thus, the adhesion is favoured when the surface free energy of both solids is lower than that of the liquid medium [4]. Therefore, it is essential to know the physicochemical interactions between the cell and the support.

The approach of van Oss et al. [5-7] considers the surface free energy of a solid or a liquid, $\gamma_{i}^{\mathrm{TOT}}$, as a sum of apolar Lifshitz-van der Waals, $\gamma_{i}^{\mathrm{LW}}$, and polar acid-base interactions, $\gamma_{i}^{\mathrm{AB}}$ :

$\gamma_{i}^{\mathrm{TOT}}=\gamma_{i}^{\mathrm{LW}}+\gamma_{i}^{\mathrm{AB}}=\gamma_{i}^{\mathrm{LW}}+2\left(\gamma_{i}^{-} \gamma_{i}^{+}\right)^{1 / 2}$

The apolar interactions consist mainly of the London dispersion interactions, but induction (Debye) and orientation (Keesom) interactions may also be involved [7]. In many cases the polar acid-base interactions are entirely due to hydrogen bonding and in the most general sense they are electron donor, $\gamma_{i}^{-}$, and electron acceptor interactions, $\gamma_{i}^{+}$. According to the authors the total interfacial free energy between phases $i$ and $j$ can be expressed as:

$$
\begin{aligned}
\gamma_{i j}^{\mathrm{TOT}}= & \gamma_{i}^{\mathrm{LW}}+\gamma_{j}^{\mathrm{LW}}-2\left(\gamma_{i}^{\mathrm{LW}} \gamma_{j}^{\mathrm{LW}}\right)^{1 / 2} \\
& +2\left[\left(\gamma_{i}^{+} \gamma_{i}^{-}\right)^{1 / 2}+\left(\gamma_{j}^{+} \gamma_{j}^{-}\right)^{1 / 2}-\left(\gamma_{i}^{+} \gamma_{j}^{-}\right)^{1 / 2}\right. \\
& \left.-\left(\gamma_{i}^{+} \gamma_{j}^{-}\right)^{1 / 2}\right]
\end{aligned}
$$

To determine the surface free energy components of a solid, contact angles of three different liquids (for which apolar and polar components are known) need to be measured. Thereafter, three forms of the following equation, resulting from Young's equation, can be solved simultaneously using the contact angle values:

$$
\begin{aligned}
W_{\mathrm{a}} & =\gamma_{1}(1+\cos \theta) \\
& =2\left(\gamma_{\mathrm{s}}^{\mathrm{LW}} \gamma_{1}^{\mathrm{LW}}\right)^{1 / 2}+2\left(\gamma_{\mathrm{s}}^{-} \gamma_{1}^{+}\right)^{1 / 2}+2\left(\gamma_{\mathrm{s}}^{+} \gamma_{1}\right)^{1 / 2}
\end{aligned}
$$

where $W_{\mathrm{a}}$ is the work of adhesion and subscript $\mathrm{s}$ and 1 mean solid and liquid, respectively. When studying interacting substances 1 and 2 that are immersed or dissolved in liquid 3, the total interaction energy, $\Delta G_{132}^{\mathrm{TOT}}$, can be expressed as:

$$
\begin{aligned}
\Delta G_{132}^{\mathrm{TOT}}= & \gamma_{12}^{\mathrm{LW}}-\gamma_{13}^{\mathrm{LW}}-\gamma_{23}^{\mathrm{LW}} \\
& +2\left[\sqrt{\gamma_{3}^{+}}\left(\sqrt{\gamma_{1}^{-}}+\sqrt{\gamma_{2}^{-}}-\sqrt{\gamma_{3}^{-}}\right)\right. \\
& +\sqrt{\gamma_{3}^{-}}\left(\sqrt{\gamma_{1}^{+}}+\sqrt{\gamma_{2}^{+}}-\sqrt{\gamma_{3}^{+}}\right) \\
& \left.-\sqrt{\gamma_{1}^{+}} \sqrt{\gamma_{2}^{-}}-\sqrt{\gamma_{1}^{-}} \sqrt{\gamma_{2}^{+}}\right]
\end{aligned}
$$

However, if a solid material is powdered it is not possible to use the contact angle technique for the surface free energy components determination. In this case, the 'thin-layer wicking' technique is of great utility [8-11]. The method is based on the phenomenon of a liquid penetration (wicking) into a solid porous layer deposited on a glass plate, e.g. microscope slide. The surface free energy components are then calculated from the proper form of the Washburn's equation, which original form reads:

$x^{2}=\frac{r t}{2 \eta} \gamma_{1 \mathrm{v}}$

where $x$ is the penetrated distance, $r$ is the capillary radius, $t$ is the penetration time of the distance $x$, $\eta$ is the liquid viscosity and $\gamma_{\mathrm{lv}}$ is the liquid surface tension. In the case of a porous layer an 'effective radius' $R$ must replace the capillary radius. However, Eq. (5) is valid only when the solid surface possesses a duplex film of the penetrating liquid ahead of the liquid front. Depending on the liquid tested and the experimental conditions chosen, four cases can be distinguished in the thin-layer wicking experiments [9-11].

(i) A low-energy liquid, for example $n$-decane, which completely wets the surface of the solid thin porous layer deposited on a glass plate. When the surface is exposed to the saturated vapour of the liquid an equilibrium state is achieved on the surface (duplex film), then Eq. (5) describes well the relationship $x^{2}=f(t)$, and the effective radius, $R$, can be determined.

(ii) The same liquid is used as above, but the plate is not exposed to the saturated vapour (bare plate). In this case the function $x^{2}=f(t)$ is described by a modified Washburn's equation [9-11].

$x^{2}=\frac{R t}{2 \eta} \Delta G_{\mathrm{b}}$ 
where $\Delta G_{\mathrm{b}}=W_{\mathrm{a}}-W_{\mathrm{c}}\left(W_{\mathrm{a}}\right.$ is the work of adhesion and $W_{\mathrm{c}}=2 \gamma_{1}$ is the work of cohesion) is the specific free energy change accompanying the penetration process.

(iii) The penetrating liquid does not completely spread onto the solid surface. A duplex film of the liquid is formed on the surface (for example by adsorption of the vapour) before the liquid penetration. The relationship $x^{2}=f(t)$ is expressed by a similar equation:

$x^{2}=\frac{R t}{2 \eta} \Delta G_{\mathrm{p}}$

where $\Delta G_{\mathrm{p}}$ now means the free energy change accompanying the penetration process along the vapor-precontacted plate.

(iv) The same liquid as in case (iii), but the thin layer of the investigated solid is not in equilibrium with the saturated vapour of the liquid. Then, the function $x^{2}=f(t)$ is again described by Eq. (6).

All the above forms of the Washburn equation should give a linear dependence of $x^{2}=f(t)$, with the slope depending on the free energy changes accompanying the liquid penetration into the porous medium.

In this study two methods were used to determine the surface tension of limestone and basalt. The aim was to compare the two methods and to correlate the free energy of adhesion of nitrifying bacteria to these two types of supporting materials.

\section{Materials and methods}

\subsection{Support materials and surface free energy determinations}

Limestone and basalt were used as the support materials (particles of $0.4-0.5 \mathrm{~mm}$ of diameter) for biomass carriers in nitrifying airlift reactors [12]. These materials are both from Portugal, regions of Porto de Mós and Mafra, respectively. Limestone has more than $90 \%$ of $\mathrm{CaCO}_{3}$ and basalt has $\sim 20 \%$ of $\mathrm{CaO}$.

As was mentioned above, the surface free energy components of these materials were determined by two techniques. The thin-layer wicking experi- ments were performed using glass plates $(25 \times 100$ $\mathrm{mm}$ ) on which a suspension of each powdered material (particle diameter $<0.38 \mu \mathrm{m}$ ) was deposited. The suspension was prepared with $5 \mathrm{~g}$ of limestone or basalt in $100 \mathrm{ml}$ of double distilled water and $5 \mathrm{ml}$ portions were pipetted onto each plate placed horizontally to obtain a uniform layer. After water evaporation (overnight) the plates were dried at $150^{\circ} \mathrm{C}$ for $2 \mathrm{~h}$ and kept in a desiccator. Some plates were allowed to equilibrate in a closed vessel with the saturated vapour of each probe liquid for $20-24 \mathrm{~h}$. To test the penetration of the liquids, the plates were placed in a Teflon chamber in a horizontal position. The chamber was $11 \mathrm{~cm}$ long, $4 \mathrm{~cm}$ wide and $0.6 \mathrm{~cm}$ deep. The top wall of the chamber was a glass plate, so the moving boundary was well visible. On the edges of the chamber, $1 \mathrm{~cm}$ sections were marked, which allowed the recording of the penetration time for particular distances ( $9 \mathrm{~cm}$ was marked). The test liquid was placed at the end of the plate with a pipette and at that very moment the penetration time counting was started. The probe liquids were decane, diiodomethane, water and formamide. In each experiment the temperature of the liquid was measured. The viscosity of the liquid was taken from literature [13]. All experiments were carried out at a room temperature of $20-22^{\circ} \mathrm{C}$. For each liquid, six plates were used, i.e. three bare and three precontacted with the liquid vapour.

Contact angle measurements were carried out at room temperature using the sessile drop technique [14] on flat plates of limestone and basalt. Water, formamide and diiodomethane were used for this purpose. These measurements ( 25 for each liquid) were performed in a standard contact angle apparatus (Krüss-Gm, Hamburg).

\subsection{Preparation of the bacteria and their surface free energy determinations}

The nitrifying consortium Nitrosomonas and $\mathrm{Ni}$ trobacter was grown in an inorganic medium: 0.85 g $\left(\mathrm{NH}_{4}\right)_{2} \mathrm{SO}_{4}, 0.25 \mathrm{~g} \mathrm{Na} \mathrm{NPO}_{4} \cdot 12 \mathrm{H}_{2} \mathrm{O}, 0.25 \mathrm{~g}$ $\mathrm{KH}_{2} \mathrm{PO}_{4}, 2.15 \mathrm{~g} \mathrm{NaHCO}_{3}, 0.015 \mathrm{~g} \mathrm{CaCl}_{2}, 0.15 \mathrm{~g}$ $\mathrm{MgSO}_{4} \cdot 7 \mathrm{H}_{2} \mathrm{O}$ and $0.005 \mathrm{~g} \mathrm{FeSO}_{4} \cdot 7 \mathrm{H}_{2} \mathrm{O}$ in 11 of distilled water, at $26^{\circ} \mathrm{C}$ in an orbital shaker (130 $\mathrm{rpm})$. The cells were harvested by centrifugation 
Table 1

Surface tension parameters of probe liquids [15], in $\mathrm{mJ} \mathrm{m}^{-2}$

\begin{tabular}{lllll}
\hline Liquid & $\gamma^{\text {TOT }}$ & $\gamma^{\text {LW }}$ & $\gamma^{+}$ & $\gamma^{-}$ \\
\hline Water & 72.8 & 21.8 & 25.5 & 25.5 \\
Glycerol & 64.0 & 34.0 & 3.9 & 57.4 \\
Diiodomethane & 50.8 & 50.8 & 0 & 0 \\
Formamide & 58.0 & 39.0 & 2.3 & 39.6 \\
$n$-Decane & 23.8 & 23.8 & 0 & 0 \\
\hline
\end{tabular}

$(10 \mathrm{~min}$ at $5000 \times g)$ in the middle exponential growth phase. The surface free energy parameters of nitrifying bacteria were determined by the contact angle measurements. The preparation of the bacteria deposited on a substratum was done according to the method of Azeredo et al. [15]. Contact angles were measured on agar plates covered with cells and using the same apparatus as above. The measurements were performed automatically with the aid of an image analysis system $(\mathrm{G} 2 / \mathrm{G} 40)$ installed in the apparatus. The images were received by a video camera connected to a 486 DX4 $100 \mathrm{MHz}$ personal computer. All the measurements were made at room temperature and the probe liquids used were water, diiodomethane and glycerol. Surface tension components of the probe liquids are listed in Table 1.

\section{Results and discussion}

While for the solid-gas interface direct surface tension measurements are possible, the determination of surface free energy of solids can only be obtained by indirect measurements. The problem of experimental and theoretical calculations of the solid surface free energy is still open. Since the approach of van Oss et al. [5-7] was published, experimental determination of apolar and polar (electron donor and electron acceptor) components of the surface free energy seems to be possible by contact angle and/or thin layer wicking techniques. The most important prerequisite for any liquid to be used in contact angle measurements is that its surface tension has to be higher than the surface free energy of the solid, otherwise the liquid would spread onto the solid surface. There is no such requirement for the liquids used in the thin layer wicking technique. However the probe liquid cannot be too viscous and volatile. The most common liquids used for this purpose are $n$-alkanes, diiodomethane, formamide and water.

The surface free energy components of Nitrosomonas and Nitrobacter bacteria are presented in Table 2, together with the measured contact angles. The components were obtained by solving Eq. (3) and using surface tension components of the liquids listed in Table 1 [16]. Analysing the results it can be found that both kinds of bacteria have the same value for the apolar component $\left(\gamma_{\mathrm{s}}^{\mathrm{LW}}=24 \mathrm{~mJ}\right.$ $\mathrm{m}^{-2}$ ), which is rather low, and they possess bipolar surface $\left(\gamma_{\mathrm{s}}^{\mathrm{AB}} \neq 0\right)$, being predominantly electron donating. The component $\gamma_{\mathrm{s}}^{-}$is much higher than $\gamma_{\mathrm{s}}^{+}$like for almost solid surfaces [17]. This polar character can result from polar groups present and/or residual water of hydration [18]. According to this notation, Nitrosomonas present a higher polar interaction than Nitrobacter $\left(\gamma_{\mathrm{s}}^{\mathrm{AB}}\right.$ equals 32.6 and $21.5 \mathrm{~mJ} \mathrm{~m}^{-2}$, respectively) although the former is less electron-donating than the latter (Table 2). The total surface free energy of Nitrosomonas is $\sim 11 \mathrm{~mJ} \mathrm{~m}{ }^{-2}$ higher than for Nitrobacter and

Table 2

Contact angles $\left(^{\circ}\right)$, surface tension components of nitrifying bacteria and the work of water spreading $W_{\mathrm{s}}$, in $\mathrm{mJ} \mathrm{m}^{-2}$

\begin{tabular}{|c|c|c|c|c|c|c|c|c|}
\hline \multirow[t]{2}{*}{ Bacteria } & \multicolumn{3}{|c|}{ Contact angle } & \multicolumn{5}{|c|}{ Free energy components } \\
\hline & $\mathrm{W}^{\mathrm{a}}$ & $G^{b}$ & $\mathrm{DM}^{\mathrm{c}}$ & $\gamma_{\mathrm{s}}^{\mathrm{LW}}$ & $\gamma_{\mathrm{s}}^{-}$ & $\gamma_{\mathrm{s}}^{+}$ & $\gamma_{\mathrm{s}}^{\mathrm{AB}}$ & $W_{\mathrm{s}}$ \\
\hline Nitrosomonas & $15.6 \pm 1.6$ & $29.4 \pm 2.0$ & $68.0 \pm 1.0$ & 24.0 & 55.2 & 4.8 & 32.6 & -2.7 \\
\hline Nitrobacter & $16.5 \pm 1.4$ & $44.6 \pm 2.1$ & $68.0 \pm 1.0$ & 24.0 & 68.9 & 1.7 & 21.5 & 3.0 \\
\hline
\end{tabular}

\footnotetext{
a Water.

b Glycerol.

${ }^{\mathrm{c}}$ Diiodomethane.
} 
Table 3

Contact angles $\left({ }^{\circ}\right)$, surface free energy components of support material and the work of water spreading $W_{\mathrm{s}}$, in $\mathrm{mJ} \mathrm{m}^{-2}$

\begin{tabular}{|c|c|c|c|c|c|c|c|c|}
\hline \multirow[t]{2}{*}{ Support material } & \multicolumn{3}{|c|}{ Contact angle } & \multicolumn{5}{|c|}{ Free energy components } \\
\hline & $\mathrm{W}^{\mathrm{a}}$ & $\mathrm{F}^{\mathrm{b}}$ & $\mathrm{DM}^{\mathrm{c}}$ & $\gamma_{\mathrm{s}}^{\mathrm{LW}}$ & $\gamma_{\mathrm{s}}^{-}$ & $\gamma_{\mathrm{s}}^{+}$ & $\gamma_{\mathrm{s}}^{\mathrm{AB}}$ & $W_{\mathrm{s}}$ \\
\hline Limestone & $51.0 \pm 2.3$ & $28.7 \pm 1.2$ & $28.5 \pm 0.4$ & 44.8 & 21.8 & 0.8 & 8.3 & -27.0 \\
\hline Basalt & $35.8 \pm 1.8$ & $31.7 \pm 1.3$ & $46.9 \pm 0.4$ & 36.0 & 41.8 & 1.1 & 13.5 & -13.8 \\
\hline
\end{tabular}

a Water.

${ }^{\mathrm{b}}$ Formamide.

${ }^{\mathrm{c}}$ Diiodomethane.

these values are typical for surfaces having intermediate free energies.

Table 3 shows the surface free energy components of limestone and basalt, obtained also by the contact angle technique. Basalt displays a higher polar component than limestone since the electron donor parameter of basalt is about twice that of limestone. This aspect reveals that the amount of water of hydration is higher for basalt.

Although the contact angle technique is a relatively simple one for solid surface free energy determination, it can only be used for plane and smooth surfaces. The method is not applicable in the case of solids in particulate form because measurements on compressed pellets rise many objections. For such solids, the thin layer wicking technique with the aid of Washburn's equation seems to be an alternative. In systems where bacteria are immobilised in particulate carriers, this technique is of great importance. Therefore the ability of both techniques for the surface free energy components determination was evaluated. The components for limestone and basalt determined by thin layer wicking are presented in Table 4. Two apolar liquids, $n$-decane and diiodomethane were independently used for apolar Lifshitz-van der Waals component determination $\left(\gamma_{\mathrm{s}}^{\mathrm{LW}}\right)$. The electron donor $\left(\gamma_{\mathrm{s}}^{-}\right)$and electron acceptor $\left(\gamma_{\mathrm{s}}^{+}\right)$parameters were determined using water and formamide. So, it was possible to conduct the calculations for two three-probe-liquid sets, namely diiodomethane-water-formamide and decane-water-formamide. Comparing the values of the components for limestone calculated from both sets of liquids (Table 4) it can be verified that they do not differ more than $2.7 \mathrm{~mJ} \mathrm{~m}^{-2}$. In the case of basalt, the differences are more significant, especially for the apolar component $\left(\gamma_{\mathrm{s}}^{\mathrm{LW}}\right)$. However, $\gamma_{\mathrm{s}}^{\mathrm{LW}}$ of basalt obtained from wicking and from contact angle (Table 3) using diiodomethane are very close. At present it is difficult to explain why the $\gamma_{\mathrm{s}}^{\mathrm{LW}}$ obtained with $n$-decane is higher. The

Table 4

Surface free energy components of support materials determined by thin layer wicking and the work of water spreading $W_{\mathrm{s}}$, in $\mathrm{mJ}$ $\mathrm{m}^{-2}$

\begin{tabular}{|c|c|c|c|c|c|c|c|c|c|c|}
\hline \multirow{2}{*}{$\begin{array}{l}\text { Support } \\
\text { Set of liquids }\end{array}$} & \multicolumn{5}{|c|}{ DM-W-F } & \multicolumn{5}{|c|}{$\mathrm{DC}-\mathrm{W}-\mathrm{F}^{\mathrm{a}}$} \\
\hline & $\gamma_{\mathrm{s}}^{\mathrm{LW}}$ & $\gamma_{\mathrm{s}}^{-}$ & $\gamma_{\mathrm{s}}^{+}$ & $\gamma_{\mathrm{s}}^{\mathrm{AB}}$ & $W_{\mathrm{s}}$ & $\gamma_{\mathrm{s}}^{\mathrm{LW}}$ & $\gamma_{\mathrm{s}}^{-}$ & $\gamma_{\mathrm{s}}^{+}$ & $\gamma_{\mathrm{s}}^{\mathrm{AB}}$ & $W_{\mathrm{s}}$ \\
\hline Limestone & 54.7 & 24.6 & 4.5 & 20.9 & -5.1 & 57.4 & 24.8 & 3.7 & 19.2 & -5.1 \\
\hline Basalt & 38.7 & 44.9 & 0.6 & 10.5 & -11.9 & 58.9 & 46.6 & 0 & 0 & -5.0 \\
\hline
\end{tabular}

\footnotetext{
${ }^{\text {a }}$ DC, decane; W, water; F, formamide.
} 
Table 5

Interfacial free energy of adhesion $\left(\Delta G_{132}\right)$ between nitrifying bacteria (1) and support materials (2), immersed in water (3), as calculated from the surface free energy components listed in Table 3 , in $\mathrm{mJ} \mathrm{m}^{-2}$, at $20^{\circ} \mathrm{C}$

\begin{tabular}{llll}
\hline System & $\Delta G_{132}^{\mathrm{LW}}$ & $\Delta G_{132}^{\mathrm{AB}}$ & $\Delta G_{132}^{\mathrm{TOT}}$ \\
\hline Nitrosomonas/water/limestone & -0.9 & 17.7 & 16.7 \\
Nitrosomonas/water/basalt & -0.6 & 27.2 & 26.6 \\
Nitrobacter/water/limestone & -0.9 & 24.2 & 23.3 \\
Nitrobacter/water/basalt & -0.6 & 36.7 & 36.1 \\
\end{tabular}

wicking times obtained with decane for bare plates were very similar (736 s \pm 23$)$, which means that the experiments were reproducible. As the polar components are concerned (Tables 3 and 4), their differences should be interpreted keeping in mind that the values obtained from contact angles deal with smooth solid surfaces and those from thin layer wicking with powdered materials. A possible explanation lays in the increase of the sites available for the interaction with the liquid, resulting from the grinding process. A similar reasoning is given by Giese et al. [19] to explain the increase in hydrophobicity of some mineral powders upon grinding. Nevertheless, even the relative values of the components are valuable for a better understanding of the adhesion process. As it can be seen from Tables 3 and 4, basalt exposes always an electron donor parameter $\left(\gamma_{\mathrm{s}}^{-}\right)$about twice that of limestone. On the other hand, from wicking method (Table 4) limestone shows a higher electron acceptor $\left(\gamma_{s}^{+}\right)$parameter than basalt. As it was mentioned above, this can be due to polar interactions, which may result from the presence of hydroxyl groups and water molecules hydrating the surface, responsible by hydrogen bondings. According to Giese et al. [19], the hydrophobicity of a solid surface can be evaluated by its $\gamma_{s}^{-}$ parameter but also remains linked to the values of $\gamma_{\mathrm{s}}^{\mathrm{LW}}$ component and the $\gamma_{\mathrm{s}}^{+}$parameter. If $\gamma_{\mathrm{s}}^{\mathrm{LW}}$ is in the range $35-45 \mathrm{~mJ} \mathrm{~m}^{-2}$, a surface having $\gamma_{\mathrm{s}}^{-}<28$ $\mathrm{mJ} \mathrm{m}^{-2}$ can be considered hydrophobic [17]. Accepting this definition, both bacteria and basalt are hydrophilic while limestone is hydrophobic (Tables 2 and 3). The same judgement may be imprecise if applied to the values obtained by the wicking technique because most of the $\gamma_{\mathrm{s}}^{\mathrm{LW}}$ values (Table 4) are out of the considered range. Another criterion for the evaluation of surface hydrophobicity is the work of water spreading, $\left(W_{\mathrm{s}}=W_{\mathrm{a}}-\right.$ $W_{\mathrm{c}}$ ), onto a solid surface. A positive value of $W_{\mathrm{s}}$ means that the liquid spreads onto the surface and no contact angle is formed. Hydrophobicity increases as the work of spreading becomes more negative. The values of the work of spreading calculated for the bacteria, limestone and basalt are listed in Tables 2-4, respectively. As it can be seen, the values of the work of spreading obtained for the support materials in plate shape (Table 3) are more negative than the ones obtained for the powdered materials (Table 4). This means that the work of adhesion in the powdered materials is higher, once more this can be due to the increase in the available sites for interaction upon grinding.

The most direct thermodynamic criterion for bacterial adhesion to a solid surface is the free energy of interaction via water phase, $\Delta G_{132}$. Tables 5 and 6 show the results of $\Delta G_{132}$, between nitrifying bacteria (1) and support materials (2), immersed in water (3), obtained by contact angle measurements and thin layer wicking, respectively.

Table 6

Interfacial free energy of adhesion $\left(\Delta G_{132}\right)$ between nitrifying bacteria (1) and support materials (2), immersed in water (3), as calculated from the components listed in Table 4 , in $\mathrm{mJ} \mathrm{m}^{-2}$, at $20^{\circ} \mathrm{C}$

\begin{tabular}{|c|c|c|c|c|c|c|}
\hline \multirow[t]{2}{*}{ System } & \multicolumn{3}{|c|}{ Water/formamide/decane } & \multicolumn{3}{|c|}{ Water/formamide/diiodomethane } \\
\hline & $\Delta G_{132}^{\mathrm{LW}}$ & $\Delta G_{132}^{\mathrm{AB}}$ & $\Delta G_{132}^{\mathrm{TOT}}$ & $\Delta G_{132}^{\mathrm{LW}}$ & $\Delta G_{132}^{\mathrm{AB}}$ & $\Delta G_{132}^{\mathrm{TOT}}$ \\
\hline Nitrosomonas/water/limestone & -1.3 & 14.5 & 13.1 & -1.3 & 13.5 & 12.2 \\
\hline Nitrosomonas/water/basalt & -1.4 & 34.2 & 32.8 & -0.7 & 29.7 & 29.0 \\
\hline Nitrobacter/water/limestone & -1.3 & 19.8 & 18.4 & -1.3 & 18.5 & 17.2 \\
\hline Nitrobacter/water/basalt & -1.4 & 46.2 & 44.8 & -0.7 & 40.1 & 39.4 \\
\hline
\end{tabular}


Despite some differences in the values of interaction energy calculated from both techniques, the resulting conclusions are the same. In all cases the adhesion of bacteria is not a thermodynamically favourable process $\left(\Delta G^{\mathrm{TOT}}>0\right)$. However, adhesion of both bacteria to limestone would be more auspicious than to basalt, because the values of the interaction energy are smaller for limestone. As it was reported [12], the nitrifying activity obtained with limestone was higher than that achieved with basalt, which implies a major colonisation of limestone particles. It should be kept in mind that other types of interaction can be present contributing for adhesion, mainly ion bonding, which are not considered in this approach.

\section{Conclusions}

The values obtained for the surface free energy components of limestone and basalt, using contact angle measurements and thin layer wicking are not exactly the same. However, for each support material, the relation between the magnitudes of the components of the surface free energy show the same trend independently of the technique used. For instance, for limestone $\gamma^{\mathrm{LW}}$ is about twice $\gamma^{-}$and for basalt $\gamma^{-}$is higher than $\gamma^{\mathrm{LW}}$.

So, despite the different values obtained by the two techniques, both enabled to obtain the same conclusions about bacterial adhesion.

\section{Acknowledgements}

The authors fully acknowledge programme
PRAXIS XXI by financial support through grant $\mathrm{BD} / 9121 / 96$ and to project 01/REGII/6/96.

\section{References}

[1] R. Oliveira, in: L.F. Melo, T.R. Bott, M. Fletcher, B. Capdeville (Eds.), Biofilms - Science and Technology (ch. 2), Kluwer, Dordrecht, 1992.

[2] A.H.L. Chamberlain, in: L.F. Melo, T.R. Bott, M. Fletcher, B. Capdeville (Eds.), Biofilms-Science and Technology (ch. 2), Kluwer, Dordrecht, 1992.

[3] P.R. Rutter, B. Vincent, in: R.C.W. Berkeley, J.M. Lynch, P.R. Rutter, B. Vincent (Eds.), Microb. Adhesion to Surfaces (ch. 4), Ellis Horwood, Chichester, 1980.

[4] N. Mozes, P.G. Rouxhet, in: L.F. Melo, T.R. Bott, M. Fletcher, B. Capdeville (Eds.), Biofilms - Science and Technology (ch. 2), Kluwer, Dordrecht, 1992.

[5] C.J. van Oss, M.K. Chaudhury, R.J. Good, Adv. Colloid Interface Sci. 28 (1987) 35.

[6] C.J. van Oss, R.J. Good, M.K. Chaudhury, Langmuir 4 (1988) 884.

[7] C.J. van Oss, L. Ju, M.K. Chaudhury, R.J. Good, J. Colloid Interface Sci. 128 (1989) 313.

[8] C.J. van Oss, Biofouling 4 (1991) 25.

[9] E. Chibowski, L. Holysz, Langmuir 8 (1993) 710.

[10] E. Chibowski, F. González-Caballero, Langmuir 9 (1993).

[11] E. Chibowski, L. Holysz, J. Adhesion Sci. Technol. 11 (1997) 1289.

[12] P. Teixeira, R. Oliveira, Bioprocess. Eng. 19 (1998) 143.

[13] D.S. Viswanath, G. Natakatan, Data Book on the Viscosity of Liquids, Hemisphere, New York, 1989.

[14] M.C.M. van Loosdrecht, J. Lyklema, W. Norde, G. Schraa, A.J.B. Zehnder, Appl. Environ. Microbiol. 53 (1987) 1893.

[15] J. Azeredo, I. Ramos, L. Rodrigues, R. Oliveira, J. Teixeira, J. Inst. Brew. 103 (1997) 359.

[16] B. Janczuk, E. Chibowski, J.M. Bruque, M.L. Kerkeb, F. Gonzalez-Caballero, J. Colloid Interface Sci. (1993) 159.

[17] C.J. van Oss, R.F. Giese, W. Wu, J. Adhesion 63 (1997) 71.

[18] C.J. van Oss, Curr. Opin. Colloid Interface Sci. 2 (1997) 503.

[19] R.F. Giese, W. Wu, C.J. van Oss, J. Dispersion Sci. Technol. 15 (1996) 527. 